\title{
May-Thurner syndrome causing deep vein thrombosis in the postoperative patient
}

\author{
Ashwini Vishalakshi L.*1, Meenakshi V. ${ }^{1}$, Amritha Ganesh ${ }^{2}$, Pallavee P. ${ }^{1}$
}

\begin{abstract}
${ }^{1}$ Department of Obstetrics and Gynaecology, ${ }^{2}$ Department of Cardiology, Mahatma Gandhi Medical College and Research Institute, Sri Balaji Vidyapeeth, Pondicherry, India
\end{abstract}

Received: 27 April 2021

Accepted: 28 May 2021

\section{*Correspondence:}

Dr. Ashwini Vishalakshi L.,

E-mail: drashwinivishal@gmail.com

Copyright: (C) the author(s), publisher and licensee Medip Academy. This is an open-access article distributed under the terms of the Creative Commons Attribution Non-Commercial License, which permits unrestricted non-commercial use, distribution, and reproduction in any medium, provided the original work is properly cited.

\begin{abstract}
May-Thurner syndrome is a rare cause of left iliac deep vein thrombosis due to an anatomical variant in which right common iliac artery overlies and compresses left common iliac vein against lumbar spine. Patients with May-Thurner syndrome usually present in their 2nd to 4th decade of life. Pregnancy and intra-abdominal surgeries are known precipitating factors in acute May-Thurner syndrome. We report the case of a woman in her 3rd decade of life who presented with May-Thurner syndrome following total abdominal hysterectomy. This case report hopes to bring attention to the high variant of patient population with May-Thurner syndrome and the necessity for surgical intervention of stent placement to prevent recurrence.
\end{abstract}

Keywords: Deep vein thrombosis, Hysterectomy, May-Thurner syndrome

\section{INTRODUCTION}

Deep vein thrombosis (DVT) is a potentially catastrophic event and one of the most dreaded complications after hysterectomy that can result in significant mortality and morbidity. The incidence of DVT in gynecologic surgery varies widely depending on the risk factors of the individual patient. About $14 \%$ of patients undergoing gynecologic surgery for benign indications develop thromboembolism. ${ }^{1}$ We report the case of a woman in her 3rd decade of life, who presented with a rare variant of deep vein thrombosis following hysterectomy.

\section{CASE REPORT}

34 years, with previous 1 lower segment cesarian section (LSCS) with body mass index (BMI) of 34 underwent total abdominal hysterectomy for adenomyosis with failed medical management. On post-operative day 9, she developed sudden onset of swelling and pain in the left lower limb. Venous Doppler of left lower limb revealed acute thrombus in the left popliteal vein, great saphenous vein and superficial femoral vein with extension up to common iliac vein. Diffuse subcutaneous edema noted from the thigh up to the ankle. Anterior tibial vein and posterior tibial vein showed flow on augmentation. Opinion was sought from the cardiologist. Considering acute onset/9 day's post-surgery/young female/ambulating patient/involvement of proximal veins with patent distal veins- May-Thurner syndrome (MTS) was suspected. Patient was started on low molecular weight heparin. Catheter directed thrombolysis was attempted but failed. Patient was started on high dose low molecular weight heparin. As the patient did not improve symptomatically and repeat Doppler showed similar findings, computed tomography (CT) venogram was done. Hypodense filling defect of 30-40 HU was seen extending from inferior vena cava (IVC) bifurcation involving left common iliac, external iliac, left common femoral vein and also extending into superficial and deep femoral veins, popliteal veins and calf veins. Thrombus was seen extending into IVC for about $12 \mathrm{~mm}$. Left common iliac vein was seen having a transverse course at the origin and was compressed between right common iliac artery and 
lumbar vertebrae. With these findings the diagnosis of MTS was made (Figure 1 and 2).
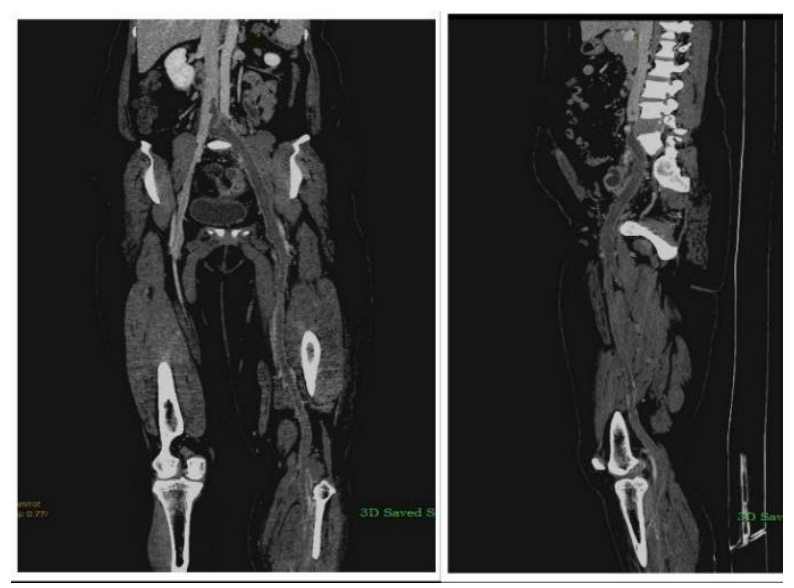

Figure 1: CT venogram-thrombus extending from IVC bifurcation involving left common iliac, external iliac, left femoral vein, popliteal vein and calf vein.

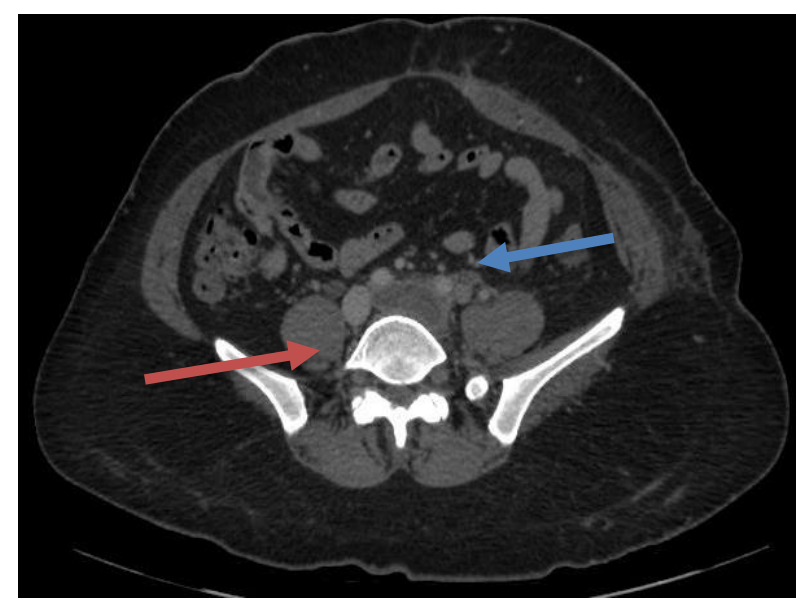

Figure 2: Red arrow- right common iliac artery, blue arrow- left common iliac vein.

Left common iliac vein venoplasty was done and the patient was started on tablet clopidogrel and tablet rivaroxaban after the procedure. Post-operative period was uneventful and the patient recovered completely on follow up.

\section{DISCUSSION}

MTS is a rare cause of left iliac deep vein thrombosis due to an anatomical variant in which, right common iliac artery overlies and compresses left common iliac vein against lumbar spine. MTS usually present in young females in their $2^{\text {nd }}$ to $4^{\text {th }}$ decades of life. Pregnancy and intra-abdominal surgeries are known precipitating factors in acute MTS. MTS was first described in 1957 by May and Thurner. According to the initial study done on 430 cadavers, this unique anatomical variant was seen in $22 \%$ of all cadavers. ${ }^{2}$
Chronic pulsatile venous compression by the right common iliac artery is responsible for the formation of intraluminal webs or spurs at the compression site. The resultant endothelial injury and turbulence in blood flow leads to thrombus formation.

Despite the relatively high incidence of this anatomical variation, the clinical prevalence of MTS- related DVT is low, reportedly occurring in only $2 \%$ to $3 \%$ of all lower extremity DVTs. ${ }^{3}$ It is thought that this low occurrence rate may be an underestimate of the actual prevalence due to missed diagnosis, the fact that there is $55.9 \%$ predominance for left sided DVT would seem to support this notion. ${ }^{4}$

Treatment is directed towards alleviating discomfort and preventing life threatening complications. Anticoagulant therapy prevents clot extension; however the existing clot and mechanical compression persist. Catheter directed thrombolysis is the most appropriate initial therapy for acute iliac vein thrombosis. Endovascular stenting obliterates spur formation and maintains long term venous patency.

\section{CONCLUSION}

MTS, a not so uncommon cause of a common condition, is an important risk factor for left leg proximal deep vein thrombosis. Of the many causes of deep vein thrombosis proposed by Virchow's triad, anatomic variants such as MTS as a cause of DVT is rarely considered. There should be a high index of suspicion of MTS in young female patients presenting with unprovoked iliofemoral DVT. This case report hopes to bring attention to MTS-a rare but potentially treatable condition which if diagnosed early can be effectively managed with thrombolysis and stent placement with good results.

\section{ACKNOWLEDGMENTS}

Authors would like to thank Department of Cardiology and Department of Radiology, MGMCRI.

\section{Funding: No funding sources \\ Conflict of interest: None declared \\ Ethical approval: Not required}

\section{REFERENCES}

1. Walsh JJ, Bonnar J, Wright FW. A study of pulmonary embolism and deep leg vein thrombosis after major gynecological surgery using labelled fibrinogen-phlebography and lung scanning. J Obstet Gynaecol Br Commonw. 1974;81(4):311-6.

2. May R, Thurner J. The cause of the predominantly sinistral occurrence of thrombosis of the pelvic veins. Angiology. 1957;8(5):419-27.

3. O'Sullivan GJ, Semba CP, Bittner CA, Kee ST, Razavi MK, Sze DY, et al. Endovascular management 
of iliac vein compression (May-Thurner) syndrome. J Vasc Interv Radiol. 2000;11(7):823-36.

4. Thijs W, Rabe KF, Rosendaal FR, Middeldorp S. Predominance of left-sided deep vein thrombosis and body weight. J Thromb Haemost. 2010;8(9):2083-4.
Cite this article as: Ashwini VL, Meenakshi V, Ganesh A, Pallavee P. May-Thurner syndrome causing deep vein thrombosis in the postoperative patient. Int J Reprod Contracept Obstet Gynecol 2021;10:2899-901. 